\section{Natural evolution from macular retinoschisis to full-thickness macular hole in highly myopic eyes}

\section{Abstract}

Purpose To evaluate the morphological changes that occurred during the development of a full-thickness macular hole (FTMH) from macular retinoschisis (MRS) in highly myopic eyes by optical coherence tomography (OCT).

Patients and methods The clinical

characteristics and OCT images of five eyes of five patients with myopic MRS who

developed FTMH during the follow-up period were evaluated.

Results The natural evolution from MRS to FTMH was classified into two patterns by OCT findings. In FTMH formation pattern 1, a focal area of the external retinal layer was elevated and followed by the development of a small outer lamellar macular hole (OLMH) and retinal detachment (RD). The OLMH and RD were then enlarged horizontally and elevated vertically until the OLMH was attached to the overlying retinal layer. A FTMH finally developed when the roof of RD opened. In FTMH formation pattern 2, the opening of the roof of MRS or cystoid space caused an inner lamellar macular hole (ILMH). The MRS was then gradually resolved except the residual MRS beneath the ILMH, the ILMH would finally proceed into a FTMH as long as the remained external retinal layer beneath the ILMH continued splitting posteriorly until it reached retinal epithelium pigment.

Conclusion Our longitudinal study revealed two patterns of FTMH formation in highly myopic eyes with MRS. This finding might be useful in further understanding the pathogenesis of macular hole formation in high myopia.

Eye (2010) 24, 1787-1791; doi:10.1038/eye.2010.123; published online 10 September 2010
CB Sun ${ }^{1}, \mathrm{Z} \mathrm{Liu}^{2}, \mathrm{AQ} \mathrm{Xue}^{3}$ and $\mathrm{K}$ Yao ${ }^{1}$

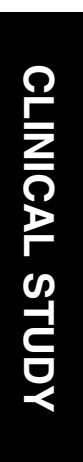

Keywords: macular hole; macular retinoschisis; optical coherence tomography; high myopia

\section{Introduction}

Macular retinoschisis (MRS) is one of the main complications leading to visual loss in highly myopic eyes. ${ }^{1-5}$ Studies have revealed that MRS could keep stable for years in some cases, however, the progression to a full-thickness macular hole (FTMH) from MRS has been frequently reported. ${ }^{1-3}$ Once a FTMH develops, the prognosis will be quite poor, for the surgical outcome of macular holes in high myopia is not satisfying. ${ }^{6}$ Unfortunately, the pathogenesis of FTMH formation from MRS is still not clear.

We evaluated the optical coherence tomography (OCT) images of five eyes of five patients followed up for at least 11 months, and revealed two patterns of FTMH formation in highly myopic eyes with MRS.

\section{Materials and methods}

The clinical data of five eyes of five patients with myopic MRS who developed a FTMH during follow-up were reviewed. Institutional review board approval was obtained. The study was conducted according to the tenets of the Declaration of Helsinki. The inclusion criteria were OCT-detected MRS without macular hole or retinal detachment (RD) at first presentation, macular hole development during follow-up, as well as myopia $\geqslant 6$ diopters and axial length $\geqslant 26 \mathrm{~mm}$. The myopic macular changes were classified into three grades according to an increasing severity as follows: mild, tigroid fundus; moderate, lacquer cracks or focal areas of deep choroidal atrophy; and severe, large
${ }^{1}$ Eye Center, Second Affiliated Hospital, School of Medicine, Zhejiang University, Hangzhou, China

${ }^{2}$ Eye Center, Zhejiang Provincial People's Hospital, Hangzhou, China

${ }^{3}$ Eye Hospital, Wenzhou Medical College, Wenzhou, China

Correspondence: K Yao, Eye Center, Second Affiliated Hospital, School of Medicine, Zhejiang

University, Hangzhou, China Tel: + 8657187783897 ;

Fax: + 8657187783897

E-mail: xlren@zju.edu.cn

Received: 30 March 2010 Accepted in revised form: 8 July 2010

Published online: 10

September 2010

The abstract of this study was accepted as an oral presentation (WE-35-08) at the XXXII International Congress of Ophthalmology in Berlin, Germany, June 2010 
geographic areas of deep choroidal atrophy ('bare sclera'). This classification was modified from the category reported by Avila et al. ${ }^{7}$ The best-corrected visual acuity (BCVA) and OCT (Stratus OCT 3, Carl Zeiss Meditec, Dublin, CA, USA) were performed every 1 to 3 months. The changes in OCT findings and BCVA among each visit of the follow-up were analysed. The procedures of OCT examination followed those reported in literature. ${ }^{3}$ The OCT scan length was $6-10 \mathrm{~mm}$, and only horizontal or vertical scans centered on the fovea were selected for evaluation. Student's $t$-test was used to compare the duration from MRS to FTMH differences between two pattern groups. A $P$-value of 0.05 was considered statistically significant.

\section{Results}

The clinical characteristics of the five eyes were shown in Table 1. At first presentation, OCT images showed MRS without RD or lamellar macular hole in all eyes, whereas foveal pseudocyst, partially detached posterior hyaloid, and epiretinal membrane were found by OCT in three, two, and one eye, respectively.

The natural evolution from MRS to FTMH was classified into two patterns by OCT findings according to whether an outer lamellar macular hole (OLMH) developed or not during a FTMH formation.

\section{FTMH formation pattern 1}

A focal area of the external retinal layer overlying retinal pigment epithelium was elevated (Figure 1a), and followed by the development of a small OLMH and a small RD (Figure 1b). The OLMH and RD were then enlarged horizontally and elevated vertically until the OLMH was attached to the overlying retinal layer (Figures 1c-e). A FTMH would finally come into being when the roof of RD opened (Figures $1 \mathrm{~d}$ and e). Occasionally, an inner lamellar macular hole (ILMH) might develop before the attachment of the OLMH to its overlying inner retinal layer and develop a FTMH (Figure 1d) (patients 1-3, Table 1).

\section{FTMH formation pattern 2}

The opening of the roof of MRS or cystoid space, if a foveal pseudocyst coexisted, caused an ILMH

(Figures 2a and b). The MRS was partially resolved after ILMH formation; however, the residual MRS beneath the ILMH could persist for a long time (Figures 2c and d). The ILMH would finally proceed into a FTMH as long as the remained external retinal tissue beneath the ILMH continued splitting posteriorly until it reached retinal epithelium pigment (Figures 2e and f) (patients 4-5, Table 1).

A dramatic decrease in BCVA was complained in all patients of pattern 1 group when an OLMH developed, although in no patients of pattern 2 group when an ILMH developed. The BCVA dropped to no more than 0.1 in all patients as soon as the FTMH developed. Vitrectomy, internal limiting membrane (ILM) peeling, and gas tamponade were performed in four patients soon after the FTMH developed. A reattached fovea was found by OCT in all cases after surgery; however, the macular hole was still open postoperatively in one patient. No other postoperative complications were found during follow-up.

The mean duration from MRS to lamellar macular hole in pattern 1 and pattern 2 group of FTMH formation was $11.3 \pm 7.0$ and $9.0 \pm 1.4$ months, respectively. The difference was not statistically significant $(P=0.689)$. However, the duration from lamellar macular hole to FTMH in pattern 1 and pattern 2 group was $4.7 \pm 2.5$ and $23.0 \pm 4.2$ months, respectively. The difference was statistically significant $(P=0.008)$.

\section{Discussion}

Macular retinoschisis is now believed to be one of the main causes of FTMH formation in high myopia. ${ }^{1-3,8,9}$

Table 1 Characteristics of five eyes of five myopic patients with full-thickness macular hole formation from macular retinoschisis

\begin{tabular}{|c|c|c|c|c|c|c|c|c|c|c|c|}
\hline \multirow[t]{2}{*}{ No. } & \multirow[t]{2}{*}{ Sex } & \multirow[t]{2}{*}{ Age } & \multirow[t]{2}{*}{$R / L$} & \multirow{2}{*}{$\begin{array}{l}\text { Refractive } \\
\text { error (D) }\end{array}$} & \multirow{2}{*}{$\begin{array}{c}\text { Axial } \\
\text { length } \\
(\mathrm{mm})\end{array}$} & \multirow{2}{*}{$\begin{array}{l}\text { Macular } \\
\text { chorioretin- } \\
\text { opathy }\end{array}$} & \multicolumn{2}{|c|}{$B C V A$} & \multirow{2}{*}{$\begin{array}{l}\text { Associated } \\
\text { OCT findings }\end{array}$} & \multicolumn{2}{|c|}{ Duration (months) } \\
\hline & & & & & & & Initial & Final & & $\begin{array}{l}\text { From } M R S \\
\text { to } L M H\end{array}$ & $\begin{array}{c}\text { From } L M H \\
\text { to FTMH }\end{array}$ \\
\hline 1 & M & 49 & $\mathrm{R}$ & -12.5 & 28.55 & Moderate & 0.8 & 0.1 & - & 4 & 7 \\
\hline 2 & $\mathrm{~F}$ & 25 & $\mathrm{R}$ & -18.5 & 30.14 & Severe & 0.2 & 0.02 & PC & 18 & 5 \\
\hline 3 & $\mathrm{~F}$ & 57 & $\mathrm{~L}$ & -11.5 & 28.04 & Severe & 0.05 & $\mathrm{CF}$ & $\mathrm{PDPH}$ & 12 & 2 \\
\hline 4 & $\mathrm{M}$ & 56 & $\mathrm{~L}$ & -16.0 & 29.40 & Moderate & 0.5 & 0.1 & PC, PDPH & 10 & 20 \\
\hline 5 & $\mathrm{M}$ & 39 & $\mathrm{R}$ & -22.5 & 31.77 & Severe & 0.3 & 0.05 & PC, ERM & 8 & 26 \\
\hline
\end{tabular}

Abbreviations: CF, counting fingers; ERM, epiretinal membrane; FTMH, full-thickness macular hole; LMH, lamellar macular hole; MRS, macular retinoschisis; PC, foveal pseudocyst; PDPH, partially-detached posterior hyaloid. 

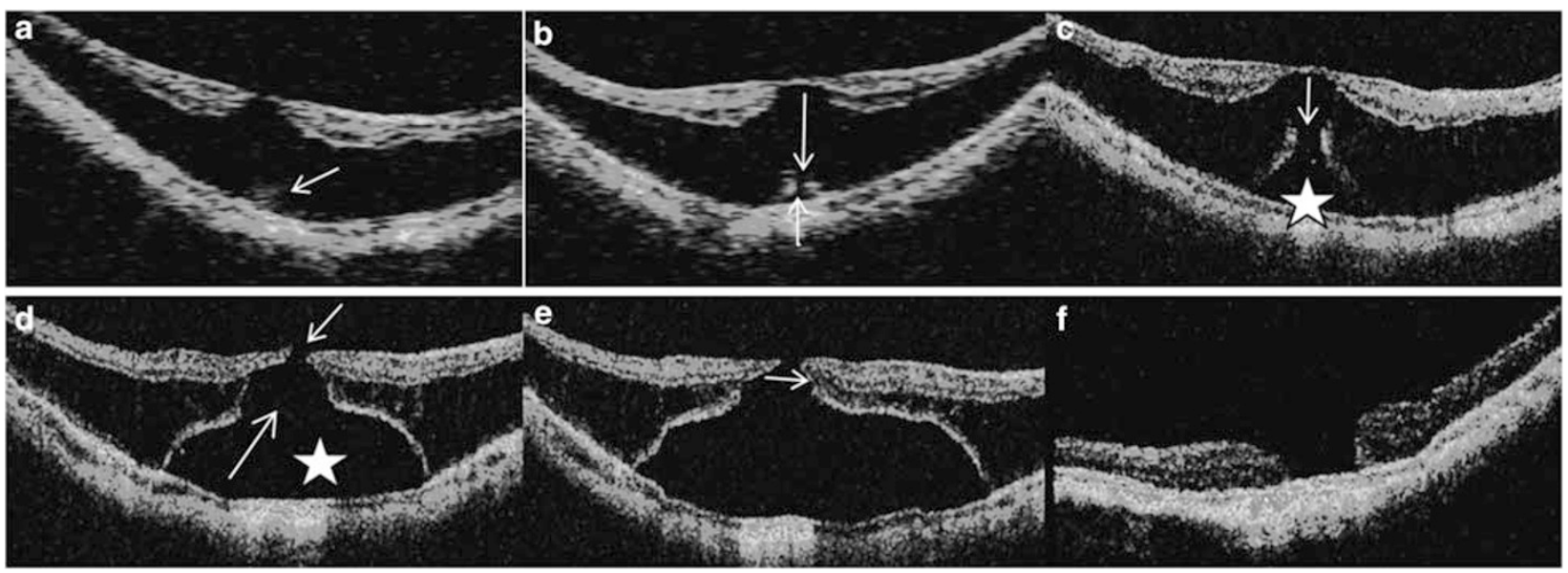

Figure 1 Development of full-thickness macular hole (FTMH) from macular retinoschisis (MRS) in case 1. (a) Optical coherence tomography (OCT) shows a MRS and focally elevated area (short arrow) of the external retinal layer. The best-corrected visual acuity (BCVA) was 0.8. (b) At 4 months later, a small outer lamellar macular hole (OLMH) (long arrow) and retinal detachment (RD) (short arrow) developed in the focally elevated area, and BCVA dropped to 0.4. Metamorphopsia appeared before OLMH development and remained until vitrectomy was performed. (c) After another 3 months, the OLMH (short arrow) and RD (star) were enlarged horizontally and elevated vertically. (d) After another 7 months of OLMH formation, OLMH (long arrow) and RD (star) continued enlarging and elevating, and FTMH developed after the rupture of the inner retina (short arrow) overlying OLMH, the BCVA dropped to 0.1. Note MRS between the inner retina and RD was still evident. (e) OCT shows FTMH and progressive enlargement and elevation of RD. Note MRS between the inner retina and RD nearly disappeared (short arrow). (f) The macular hole was still open after vitrectomy, even though metamorphopsia regressed.
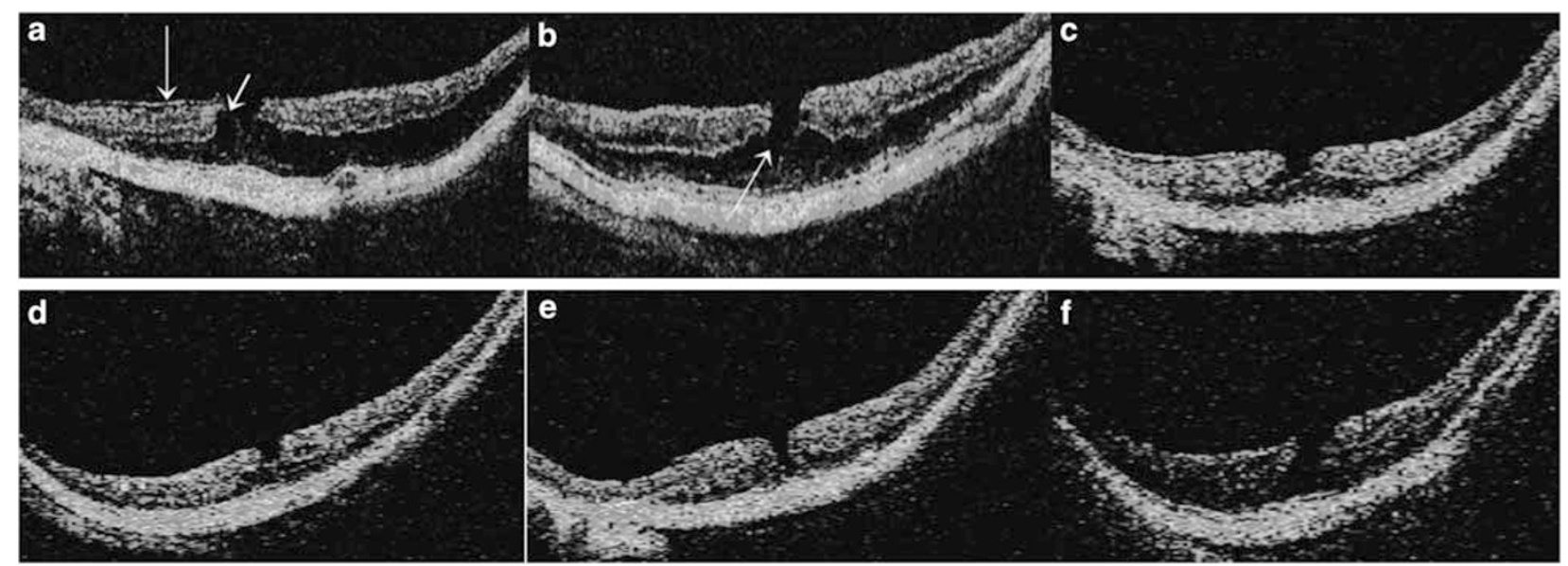

Figure 2 Development of full-thickness macular hole (FTMH) from macular retinoschisis (MRS) without outer lamellar macular hole (OLMH) formation in case 5. (a) OCT shows MRS was accompanied by foveal pseudocysts (short arrow) and epiretinal membrane (long arrow). (b) At 8 months later, the roof of a foveal pseudocyst opened (short arrow) and an inner lamellar macular hole (ILMH) developed, BCVA decreased from 0.3 to 0.2. (c and d) The MRS was mostly resolved except the residual MRS beneath the ILMH. (e) The remained external retinal tissue beneath the ILMH continued splitting posteriorly. (f) After another 26 months of ILMH formation, FTMH finally developed and BCVA dropped to 0.05.

However, much more study has to be done to make clear the pathogenesis of FTMH formation from MRS. In our study, two different patterns of progression from MRS to FTMH have been revealed by OCT. Many elements could contribute to the development of FTMH in MRS cases. First, the focal elevation of the external retinal layer and the following development of OLMH were probably because of the increased inward traction, which was caused by the rigidity of the ILM and inflexibility of the retinal vessel, and then transferred by the column-like structures within MRS. ${ }^{10-14}$ Second, premacular structures, such as partially detached posterior hyaloid and epiretinal membrane, could cause a tangential traction to fovea and facilitate to develop a FTMH or 
ILMH in myopic eyes with MRS, as they did in idiopathic macular hole formation in emmetropic eyes. ${ }^{15,16}$ Furthermore, premacular structures could also facilitate the development of OLMH in pattern 1 group, as well as posteriorly splitting and horizontal enlargement of the external retinal layer in pattern 2 group by transmitting their traction from ILM to photoreceptor layer, for most cone pedicles in the fovea were sheathed by Müller cells. ${ }^{14}$ Third, the persistent vitreomacular traction might enhance the myopic macular degeneration, which might proceed the formation of OLMH or cystoid spaces and finally lead to FTMH. Macular architecture might be modified because of cell loss and not only because of traction. ${ }^{17,18}$

The natural evolution from MRS into FTMH has not been well described. Gaucher et al ${ }^{1}$ revealed FTMH was apt to occur in MRS cases with RD by OCT after long-term follow-up. Our study confirmed that RD was an intermediate-stage change in the development of FTMH in some MRS cases, even though MRS and RD could coexist for a long period. ${ }^{1,8}$ Many previous studies have shown that ILMH was accompanied with MRS in some cases. In our study, ILMH developed and finally proceeded into FTMH in three highly myopic eyes with MRS, just as in non-highly myopic eyes. ${ }^{15,16}$

In our study, the mean duration from MRS to lamellar macular hole in pattern 1 and pattern 2 group of FTMH formation was similar. However, the duration from lamellar macular hole to FTMH in pattern 2 group was much longer than in pattern 1 , which might be explained by the significant resolution in both inward traction and the traction of premacular structures after the ILM was broken in pattern 2 group.

Although surgical intervention, including vitrectomy, ILM peeling, and gas tamponade, has proven to be effective and safe in the treatment of most MRS cases, rare yet severe postoperative complications, such as FTMH formation, do occur in a few MRS cases. ${ }^{1,6,8,19}$ Furthermore, considering some MRS could keep stable for years, more clinical studies are needed to make clear when is the proper time and which are the best techniques for the surgical intervention of MRS. ${ }^{1,6,8,19-21}$ Our longitudinal study indicated that FTMH might finally develop if MRS was accompanied by foveal detachment or premacular structures. This finding further supported the hypothesis that premacular structures and foveal detachment were two risk factors for a poor prognosis in MRS patients. ${ }^{1}$ Therefore, it might be a reasonable choice to perform vitrectomy in MRS cases accompanied by premacular structures and (or) foveal detachment.

In conclusion, our longitudinal study revealed two patterns of FTMH formation in highly myopic eyes with MRS, inward and vitreomacular traction were hypothesized to be the main cause of FTMH formation in these cases.

\section{Summary}

\section{What was known before}

- Macular retinoschisis is one of the main complications leading to visual loss in highly myopic eyes, for it has a high risk to progress to a full-thickness macular hole with a poor prognosis. Unfortunately, the pathogenesis of macular hole formation from macular retinoschisis is still not clear.

What this study adds

- To our knowledge, this is the first longitudinal, observational study, which provides different patterns of full-thickness macular hole formation from macular retinoschisis in literature.

\section{Conflict of interest}

The authors declare no conflict of interest.

\section{References}

1 Gaucher D, Haouchine B, Tadayoni R, Massin P, Erginay A, Benhamou $\mathrm{N}$ et al Long-term follow-up of high myopic foveoschisis: natural course and surgical outcome. Am J Ophthalmol 2007; 143: 455-462.

2 Shimada N, Ohno-Matsui K, Baba T, Futagami S, Tokoro T, Mochizuki M. Natural course of macular retinoschisis in highly myopic eyes without macular hole or retinal detachment. Am J Ophthalmol 2006; 142: 497-500.

3 Benhamou N, Massin P, Haouchine B, Erginay A, Gaudric A. Macular retinoschisis in highly myopic eyes. Am J Ophthalmol 2002; 133: 794-800.

4 Baba T, Ohno-Matsui K, Futagami S, Yoshida T, Yasuzumi $\mathrm{K}$, Kojima A et al Prevalence and characteristics of foveal retinal detachment without macular hole in high myopia. Am J Ophthalmol 2003; 135: 338-342.

5 Sun CB, Wang QM, Xue AQ, Chen B, Huang J, Sun T. Macular retinoschisis and retinal detachment without hole in high myopia (in Chinese). Chin J Ocul Fundus Dis 2009; 25: 327-330.

6 Ikuno Y, Tano Y. Vitrectomy for macular holes associated with myopic foveoschisis. Am J Ophthalmol 2006; 141: 774-776.

7 Avila MP, Weiter JJ, Jalkh AE, Trempe CL, Pruett RC, Schepens CL. Natural history of choroidal neovascularization in degenerative myopia. Ophthalmology 1984; 91: 1573-1581.

8 Ikuno Y, Sayanagi K, Soga K, Oshima Y, Ohji M, Tano Y. Foveal anatomical status and surgical results in vitrectomy for myopic foveoschisis. Jpn J Ophthalmol 2008; 52: 269-276.

9 Takano M, Kishi S. Foveal retinoschisis and retinal detachment in severely myopic eyes with posterior staphyloma. Am J Ophthalmol 1999; 128: 472-476.

10 Shimada N, Ohno-Matsui K, Yoshida T, Sugamoto Y, Tokoro T, Mochizuki M. Progression from macular retinoschisis to retinal detachment in highly myopic eyes is associated with outer lamellar hole formation. Br J Ophthalmol 2008; 92: 762-764. 
11 Sayanagi K, Ikuno Y, Tano Y. Tractional internal limiting membrane detachment in highly myopic eyes. Am J Ophthalmol 2006; 142: 850-852.

12 Sayanagi K, Ikuno Y, Gomi F, Tano Y. Retinal vascular microfolds in highly myopic eyes. Am J Ophthalmol 2005; 139: 658-663.

13 Ikuno Y, Gomi F, Tano Y. Potent retinal arteriolar traction as a possible cause of myopic foveoschisis. Am J Ophthalmol 2005; 139: 462-467.

14 Gaudric A. Macular cysts, holes and cavitations: 2006 Jules Gonin lecture of the Retina Research Foundation. Graefes Arch Clin Exp Ophthalmol 2008; 246: 1071-1079.

15 Judson PH, Yannuzzi LA. Macular hole. In: Ryan SJ, Schachat AP, Murphy RP (eds.) Retina 2nd ed. Vol. 2. Medical Retina. Mosby: St. Louis, 1994; 1169-1185.

16 Takahashi H, Kishi S. Tomographic features of a lamellar macular hole formation and a lamellar hole that progressed to a full-thickness macular hole. Am J Ophthalmol 2000; 130: 677-679.
17 Sayanagi K, Ikuno Y, Soga K, Tano Y. Photoreceptor inner and outer segment defects in myopic foveoschisis. Am J Ophthalmol 2008; 145: 902-908.

18 Shimada N, Ohno-Matsui K, Nishimuta A, Nishimuta A, Moriyama M, Yoshida $\mathrm{T}$ et al Detection of paravascular lamellar holes and other paravascular abnormalities by optical coherence tomography in eyes with high myopia. Ophthalmology 2008; 115: 708-717.

19 Kwok AK, Lai TY, Yip WW. Vitrectomy and gas tamponade without internal limiting membrane peeling for myopic foveoschisis. Br J Ophthalmol 2005; 89: 1180-1183.

20 Sun $\mathrm{CB}$, Xue AQ, Yao K. Is posterior scleral reinforcement sufficient for the treatment of myopic foveoschisis? Clin Experiment Ophthalmol 2010; 38: 429-430.

21 Baba T, Tanaka S, Maesawa A, Teramatsu T, Noda Y, Yamamoto S. Scleral buckling with macular plombe for eyes with myopic macular retinoschisis and retinal detachment without macular hole. Am J Ophthalmol 2006; 142: $483-487$. 